Revue internationale P.M.E.

Économie et gestion de la petite et moyenne entreprise

\title{
Réseaux d'innovation dans les PME en estrie
}

\section{David Doloreux et Yannik Melançon}

Volume 20, numéro 1, 2007

URI : https://id.erudit.org/iderudit/1008510ar

DOI : https://doi.org/10.7202/1008510ar

Aller au sommaire du numéro

Éditeur(s)

Presses de l’Université du Québec

ISSN

0776-5436 (imprimé)

1918-9699 (numérique)

Découvrir la revue

Citer cet article

Doloreux, D. \& Melançon, Y. (2007). Réseaux d'innovation dans les PME en estrie. Revue internationale P.M.E., 20(1), 69-90.

https://doi.org/10.7202/1008510ar
Résumé de l'article

À partir des données provenant d'une enquête réalisée auprès de 50 petites et moyennes entreprises de la région de l'Estrie au Québec, cet article cherche à comprendre et décrire les activités d'innovation et à analyser les formes de collaboration de provenance régionale, nationale et internationale. Au terme de l'analyse empirique, les résultats démontrent différents niveaux d'interactions spatiales liés à l'innovation et mettent en valeur à la fois l'importance des ressources régionales et celle des réseaux plus larges. Des résultats empiriques de l'étude remettent en question certaines prémisses liées à la dimension régionale de l'innovation. 


\title{
Réseaux d'innovation dans les PME en Estrie
}

\author{
David DOLOREUX \\ Chaire de recherche sur la francophonie canadienne \\ Université d'Ottawa \\ Yannik MELANÇON \\ Département des sciences humaines \\ Université du Québec à Rimouski
}

\section{MOTS CLÉS}

\section{Innovation - Réseaux d'innovation - PME}

Système régional d'innovation - Estrie

\begin{abstract}
LES AUTEURS
DAVID DOLOREUX est professeur agrégé à la Faculté de gestion de l'Université d'Ottawa et titulaire de la Chaire de recherche en francophonie canadienne sur l'entrepreneuriat, l'innovation et le développement régional. Ses travaux portent sur divers sujets, dont les impacts des développements récents dans le domaine de l'innovation et de l'apprentissage sur les économies régionales au Canada et ailleurs en Europe. II travaille actuellement sur l'innovation dans les entreprises de services dans les régions canadiennes et européennes. Adresse: Chaire de recherche en francophonie canadienne, School of Management, Université d'Ottawa, 136, rue Jean-Jacques-Lussier, Ottawa, Ontario, K1N 6N5. Courriel : <doloreux@management. uottawa.ca>.
\end{abstract}

YANNIK MELANÇON est étudiant au doctorat en développement régional à l'Université du Québec à Rimouski. Ses travaux de recherche portent sur l'innovation et le développement régional. Il a aussi œuvré à titre de coordonnateur de l'Observatoire sur le système régional d'innovation de l'Estrie, un partenaire dans la réalisation de l'étude dont les résultats sont présentés ici. Ses travaux actuels portent sur le développement de l'industrie maritime au Québec et sur les systèmes de soutien à l'innovation. Adresse: Département des sciences humaines, Université du Québec à Rimouski, 300, allée des Ursulines, Rimouski, Québec, G5L 3A1. Courriel <yannik.melancon@uqar.qc.ca>.

\section{RÉSUMÉ}

À partir des données provenant d'une enquête réalisée auprès de 50 petites et moyennes entreprises de la région de l'Estrie au Québec, cet article cherche à comprendre et décrire les activités d'innovation et à analyser les formes de collaboration de provenance régionale, nationale et internationale. Au terme de l'analyse empirique, les résultats démontrent différents niveaux d'interactions spatiales liés 
à l'innovation et mettent en valeur à la fois l'importance des ressources régionales et celle des réseaux plus larges. Des résultats empiriques de l'étude remettent en question certaines prémisses liées à la dimension régionale de l'innovation.

\begin{abstract}
Based on a survey carried out on 50 small and medium-sized enterprises in the Estrie region, in Quebec, the article seeks to better understand the innovation activities and collaborations initiated at the local, national and international levels. At the end of the empirical analysis, the results show various geographical levels of networks related to innovation, emphasizing both the relative importance of the regional and international environments. Some of the results of the study call into question certain premises related to the regional dimension of innovation.
\end{abstract}

\title{
RESUMEN
}

Este artículo intenta explicar y describir las actividades de innovación así como analizar las formas de colaboración regional, provincial, nacional e internacional, a partir de datos provenientes de una encuesta realizada en 50 pequeñas y medianas empresas de la región de Estrie (Quebec). Al término del análisis empírico, los resultados demuestran distintos niveles de interacciones espaciales ligadas a la innovación resaltan la importancia tanto de los recursos regionales y como de redes más amplias. Algunos resultados empíricos del estudio cuestionan ciertas premisas ligadas a la dimensión regional de la innovación.

\section{ZUSAMMENFASSUNG}

Anhand von Daten aus einer Untersuchung von 50 kleinen und mittleren Unternehmen der Region Estrie in Quebec beschreibt der vorliegende Artikel die Innovationsaktivitäten dieser Unternehmen und analysiert die verschiedenen Formen der Zusammenarbeit auf regionaler, nationaler und internationaler Ebene.

Durch die empirische Analyse konnte aufgezeigt werden, dass verschiedene räumliche Interaktionen im Zusammenhang mit Innovation bestehen und wie wichtig dabei die regionalen und überregionalen Ressourcen sind. Die Studie stellt somit gewisse Prämissen im Zusammenhang mit der regionalen Dimension von Innovation in Frage.

\section{Introduction}

La nouvelle conceptualisation de l'innovation met en évidence le rôle de la proximité dans les processus d'innovation. En partant de l'hypothèse voulant que la proximité géographique facilite les rapports entre l'entreprise innovante et les apports externes qui lui sont nécessaires lors du processus d'innovation, plusieurs chercheurs ont récemment interrogé le rôle de l'en- 
vironnement régional et de la proximité géographique dans ce processus (Doloreux, 2004a, 2004b; Simmie, 2003; Freel, 2002, 2003 ; Edquist, Eriksson et Sjören, 2000, 2002; Diez, 2000; Koschatzky, Kulicke et Zenker, 2001; Cooke, Boekholt et Tödtling, 2000; Henry et Pinch, 2000; Sternberg, 2000). Or, des travaux récents portant sur le transfert et le partage des connaissances révèlent l'importance des relations extraterritoriales dans les processus d'innovation des entreprises (MacKinnon, Cumbers et Chapman, 2002; MacKinnon, Chapman et Cumbers, 2004; Simmie, 2001, 2003 ; Britton, 2003, 2004 ; Bunnel et Coe, 2001). Ces travaux ont démontré à partir de différentes études de cas que les entreprises innovantes utilisent des connaissances qui proviennent de multiples sources régionales, nationales et internationales.

À partir des données provenant d'une enquête réalisée auprès de 50 petites et moyennes entreprises (PME) de la région de l'Estrie au Québec, cet article poursuit deux objectifs:

- relever les pratiques innovantes des PME et les mécanismes leur permettant d'adopter des formes de production innovante;

- définir les formes de collaboration de provenance régionale, nationale et internationale et, le cas échéant, les types de relations que les entreprises entretiennent avec le système régional et le système national d'innovation.

Pour apporter des éléments de réponse à ces deux objectifs, nous proposons, à la section suivante, une réflexion sur les fondements conceptuels du rôle de la région et de la proximité dans les réseaux d'innovation. La troisième section traite de la méthodologie et de l'enquête, alors que la quatrième section présente le profil socioéconomique de la région de l'Estrie. La cinquième partie s'intéresse aux résultats empiriques et examine plus précisément les pratiques innovantes des entreprises et les réseaux d'innovation. La conclusion discute des résultats de l'étude et de l'échelle géographique à laquelle opèrent les réseaux d'innovation des entreprises de l'Estrie.

\section{Réseaux d'innovation et régions}

Les entreprises n'innovent pas en vase clos; l'innovation est un processus fondé sur des relations de proximité et, par conséquent, elle est intimement influencée et stimulée par l'environnement socioéconomique. Celui-ci, s'il réunit les conditions favorables, peut permettre d'accroître la capacité d'apprentissage interactif en facilitant les rapports entre l'entreprise et les apports externes qui lui sont nécessaires pour innover. Autrement dit, la région offre 
et produit les conditions et les ressources nécessaires à l'innovation, alors que celle-ci est à la base du développement et de la croissance. Cette section propose une réflexion théorique sur les réseaux d'innovation et les régions.

\subsection{Réseaux d'innovation}

Plutôt que d'aborder l'innovation comme le résultat d'un processus linéaire et technocratique et la région comme un simple support à l'affectation des ressources, les travaux récents sur l'innovation présentent celle-ci comme étant le résultat d'interactions multiples entre l'entreprise et divers agents de son environnement, par exemple, les fournisseurs, les clients, les organisations professionnelles et les institutions de formation et gouvernementales (Asheim et Gertler, 2005). Ainsi, l'innovation est conceptualisée comme un processus itératif qui implique des formes collectives d'apprentissage facilitant l'accès à différentes sources d'information pour l'entreprise (Edquist, 1997). C'est à travers ses réseaux que l'entreprise accède aux diverses sources d'information dont il est question ici. Les réseaux jouent donc un rôle déterminant dans la capacité d'innovation des entreprises.

L'approche des systèmes d'innovation accorde une place centrale à la question des réseaux d'innovation et la littérature offre plusieurs définitions des réseaux d'innovation (De Bresson et Amesse, 1991; Tijssen, 1998) ou de concepts similaires, par exemple, l'apprentissage interactif et collectif. Tijssen (1998, p. 72) définit un réseau comme étant «Un système d'interdépendance évolutif basé sur des relations dont le caractère systémique est le résultat d'interactions, de procédés, de procédures et d'institutionnalisation. Les activités dans un tel réseau mettent à contribution la création, la combinaison, l'échange, la transformation, l'absorption et l'exploitation de ressources à l'intérieur d'une gamme de relations formelles et informelles ${ }^{1} »$.

Les réseaux d'innovation se déclinent en de multiples variantes. Les motivations et objectifs derrière la création de ces réseaux déterminent leur nature alors que leur contenu et leur forme sont fonction des différents acteurs concernés (Fischer 2001). À une extrémité du spectre, il y a les relations hautement formalisées, par exemple, les liens verticaux qui unissent les utilisateurs et les producteurs. Ces liens servent tant à réduire les coûts de transaction associés à l'échange d'information qu'à diminuer les risques associés à l'introduction de nouveaux produits sur le marché. À l'autre

1. Traduction libre de «An evolving mutual dependency system based on resource relationships in which their systemic character is the outcome of interactions, processes, procedures and institutionalization. Activities within such a network involve the creation, combination, exchange, transformation, absorption and exploitation of resources within a range of formal and informal relationships. » 
extrémité du spectre, il y a les relations informelles qui lient les acteurs au sein de chaînes ouvertes. Ainsi, les réseaux horizontaux unissent les individus et les organisations œuvrant dans certaines fonctions spécifiques, comme la recherche, la production, la logistique ou le marketing.

Pour Tödtling et Kaufman (2001), l'intégration des PME au sein de réseaux et systèmes d'innovation constitue une manière de contourner les limites associées à leur taille aux compétences internes limitées qui y sont associées. Pour Romijn et Albaladejo (2002), la mobilisation de ressources externes permet d'augmenter le caractère innovant de l'entreprise. Par ailleurs, pour les $\mathrm{PME}$, les réseaux représentent une réponse à l'insécurité et à l'incertitude associées à l'innovation, que ce soit dans le développement de nouveaux produits ou dans l'utilisation de nouvelles technologies (Rogers, 2004).

\subsection{Réseaux d'innovation, proximité et régions}

Les échanges d'information sont au cœur des activités qui animent les réseaux d'innovation. La proximité sociale, la proximité culturelle et la proximité géographique sont toutes des conditions de départ nécessaires à l'instauration de ces échanges informationnels (Koschatzky, Kulicke et Zenker, 2001). La littérature traitant de l'importance de la proximité dans l'innovation est abondante. Dans l'approche des districts industriels, les PME tirent avantage de réseaux locaux et régionaux basés sur la confiance et profitent d'institutions communes dans les systèmes locaux de production (Camagni, Maillat et Matteaccioli, 2004). L'approche du milieu innovateur présente une vision similaire, en ce sens qu'elle met l'accent sur le rôle des aspects sociaux et culturels dans l'élaboration d'un climat de confiance et de réciprocité crucial pour la diffusion des formes de savoir (Maillat et Kébir, 2001). Dans l'approche des régions apprenantes, les différentes combinaisons de proximité sociale, culturelle et spatiale permettent aux entreprises de mettre en place les conditions nécessaires pour réduire l'incertitude économique et technologique associée à l'innovation (Morgan, 1997). Finalement, le concept du système régional d'innovation stipule que la proximité favorise les formes d'apprentissage interactif liant les entreprises avec l'ensemble des acteurs de leur environnement socioéconomique (Doloreux, 2004a).

La concentration et la proximité géographique des acteurs procurent des avantages indéniables aux entreprises en permettant à celles-ci d'accéder aux ressources complémentaires dont elles ont besoin pour se développer, tant économiquement que technologiquement. En effet, selon Malmberg et Maskell (1997), l'accès aux marchés, aux fournisseurs, à une main-d'œuvre qualifiée et possédant des compétences variées, l'accès aux réseaux formels 
et informels, aux services aux entreprises spécialisés et à une infrastructure technologique de qualité sont des ressources importantes pour l'entreprise et particulièrement pour l'entreprise innovante, car leur prospérité dépend de leur capacité de collecter et de traiter de l'information, de répondre rapidement à la demande et d'intégrer les intrants provenant d'autres organisations présentes au sein de leur environnement.

De plus, certaines régions, en plus de bénéficier de la convergence des facteurs économiques énoncés précédemment, jouissent d'un cadre institutionnel et social qui leur permet d'attirer, de créer et de diffuser de l'information et des connaissances. Malgré les fluctuations économiques, ces régions tendent à maintenir leurs avantages une fois ceux-ci acquis.

Bien que la proximité physique et les forces d'agglomération jouent un rôle important, d'autres formes de proximité doivent être présentes pour permettre aux réseaux régionaux d'innovation d'émerger (Gregersen et Johnson, 1997). En effet, la proximité spatiale des réseaux d'innovation doit s'insérer dans une vision sociale et culturelle partagée, car sans celle-ci, le développement de relations étroites entre les agents peut être entravé (Lundvall, 1992).

Les partisans de l'approche de l'encastrement social maintiennent quant à eux que les réseaux régionaux sont influencés par les structures sociales (Grabher, 1993). S'inscrivant dans cette perspective, Lyons (2000) écrit que «Il est attendu que l'encastrement social des firmes renforce le milieu en développant un sens industriel commun et un consensus social; en offrant des manières communes de percevoir les problèmes économiques et techniques, de même que leur solution; et en favorisant le développement d'un support institutionnel étendu et d'un support informel encourageant l'innovation, le

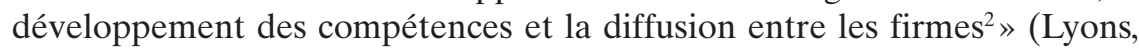
2000, p. 893).

Lorenzen (1998) abonde dans ce sens et examine l'importance de la proximité dans le réseautage «Les agglomérations géographiques de firmes localisées dans une même région, comme dans les districts industriels, et faisant partie d'une communauté d'entreprises partageant un environnement socioéconomique [...] peuvent favoriser le partage des connaissances tacites. La proximité est aussi importante en ce qui a trait aux canaux de

2. Traduction libre de «Social embeddedness of firms is expected to strengthen the milieu by developing a sense of common industrial purpose and social consensus; common ways of perceiving economic and technical problems and solutions; and the development of extensive institutional and informal support that encourage innovation, skill formation, and the circulation among the firms. » 
communications concernés, car ces canaux, au sein desquels l'information est partagée comme dans les sports de contact, constituent un lien physique entre les gens ${ }^{3} »$ (Lorenzen, 1998, p. 154-155).

Puisque les échanges d'information au sein de réseaux, conditions nécessaires à l'innovation, nécessitent la combinaison de diverses proximités (géographique, culturelle, sociale) pour se développer, certains auteurs expliquent que les réseaux d'innovation sont créés et maintenus à travers des processus et mécanismes hautement localisés (Maskell, 2001; Maskell et Malberg, 1999; Lorenzen, 1998; Porter, 1998; Storper, 1997). S’il en est ainsi, c'est que le savoir tacite, les compétences de la main-d'œuvre et la capacité de manipuler les savoirs codifiés sont incorporés et fonction du contexte spécifique et, conséquemment, transférables uniquement grâce à certains types d'interactions sociales. Pour arriver à transmettre ces savoirs tacites, un haut degré de confiance entre les organisations est requis (Maskell et Malberg, 1999).

Ainsi, la confiance joue un rôle crucial dans le développement des réseaux d'innovation. Celle-ci doit s'établir tant au plan personnel que social et elle est renforcée par le partage et l'utilisation entre les acteurs d'un langage, de valeurs et d'une culture commune. Elle doit donc être bâtie grâce à un système social, ce qui implique un processus évolutif qui demande du temps (Amable, Barré et Boyer Amable, 1997). De ce point de vue, l'encastrement émerge comme un processus territorialisé, avec une concentration significative d'entreprises et d'institutions partageant des valeurs au plan social et culturel et diverses ressources qui peuvent être utilisées pour engendrer de nouveaux produits et processus. En règle générale, la géographie joue un rôle fondamental dans le processus d'innovation et d'apprentissage, car les innovations sont moins le produit d'une seule entreprise que l'assemblage des ressources, connaissances et capacités qui sont localisées dans des endroits spécifiques (Maskell et Malberg, 1999).

Cela dit, des travaux récents portant sur le transfert et le partage des connaissances révèlent l'importance des relations extraterritoriales dans les processus d'innovation des entreprises (Bunnell et Coe, 2001; Doloreux, 2004a, 2004b; Simmie, 2001, 2003; Britton, 2003, 2004; MacKinnon, Cumbers et Chapman, 2002). Ces travaux ont questionné la véritable nature de

3. Traduction libre de « Geographical agglomerations of firms, as in industrial districts, located in the same region and being communities of firms sharing a common socio-economic environment [and thus conception of what has prominence] can be beneficial to sharing tacit knowledge. Proximity also matters when information channels are concerned, because the channels, through which much information is shared as body-to-body contact sport, played through physical contact between people.» 
l'ancrage territorial des processus d'innovation et, par conséquent, le rôle de région dans les réseaux d'innovation. De telles critiques sont étroitement liées au fait que les entreprises innovantes exploitent à la fois des ressources endogènes et exogènes qui, mises ensemble, leur confèrent des avantages concurrentiels leur permettant d'innover et de renforcer leur intégration dans les marchés. Il en va de même pour les dispositifs mis en œuvre dans une optique de construction territoriale du système d'innovation, qui reposent plus précisément sur des interventions publiques de niveaux spatiaux variés (Howells, 1999).

Une étude portant sur des entreprises à Ottawa et en Beauce illustre que, dans le processus d'innovation, les PME qui exploitaient des ressources locales et internationales détenaient un avantage concurrentiel en comparaison des entreprises qui se basaient uniquement sur les ressources locales pour innover (Doloreux, 2004a; 2004b; 2003). Simmie (2003), quant à lui, a démontré, dans le cadre d'une étude en Europe, l'importance pour les entreprises innovantes des transferts et partages de connaissance, tant à l'intérieur de la région qu'avec des entreprises provenant de régions similaires dans l'économie internationale. Bitard et Doloreux (2006) précisent, dans le contexte de la Suède, que les entreprises innovantes accordent autant d'importance aux sources d'information localisées et à celles plus diffuses, et ce, indépendamment de leur provenance géographique. Ces observations sont semblables à celles réalisées par Larsson et Malmberg (1999) qui ont conclu dans leur étude sur l'industrie suédoise de la machinerie que le réseautage local direct n'avait pas d'incidence sur la performance des entreprises. Suarlez-Villa et Walrod (1997) ont, pour leur part, affirmé que la concentration géographique des industries électroniques en Californie n'avait pas entraîné de plus grandes opportunités d'innovation pour ces entreprises, des résultats semblables à ceux de Britton (2003) qui a démontré l'importance des liens internationaux dans les processus d'innovation des industries électroniques à Toronto.

\section{Méthodologie}

L'étude réalisée poursuit le double objectif de mieux comprendre les capacités innovantes des entreprises et les formes de collaboration et d'interaction des entreprises lors des activités d'innovation.

Pour répondre à ces objectifs, nous avons conduit une enquête auprès des dirigeants d'entreprises de la région de l'Estrie au Québec entre le 4 février et le 7 avril 2005. L'enquête a consisté à interroger les dirigeants en leur soumettant un questionnaire électronique administré par Internet. La population ciblée par cette enquête est composée de PME ayant moins de 
200 employés en Estrie, œuvrant dans le secteur de la fabrication (SCIAN 31 à 33) ou dans le domaine des services scientifiques et techniques (SCIAN 541) et ayant demandé, pendant la période 2002 à 2004, des crédits d'impôt pour leurs activités de R-D ${ }^{4}$.

L'échantillon de notre enquête a été construit à partir de différents répertoires industriels, tels que la «Liste des commerces et industries de l'Estrie», le «Répertoire de la R-D industrielle» et le répertoire des «Entreprises innovatrices du secteur de la santé». Au total, 222 entreprises ont été sélectionnées et 50 d'entre elles ont participé à l'enquête, pour un taux de réponse de $22,5 \%$. Ce taux de réponse est comparable à d'autres études qui ont analysé les réseaux d'innovation des PME (Doloreux, 2004a; Britton, 2004; Romijn et Albaladejo, 2002; Freel, 2002).

Le questionnaire réalisé aux fins de cette enquête est inspiré des principes énoncés dans le manuel d'Oslo de l'OCDE (1997) et des enquêtes nationales sur l'innovation découlant de l'application de ces principes, notamment les Community Innovation Survey et les Enquêtes sur l'innovation de Statistique Canada.

Le tableau 1 donne une vue d'ensemble de l'échantillon. En ce qui a trait à la période de création des entreprises, 62,0\% des entreprises ont été créées après 1990 et 24,0 \% des entreprises avaient moins de cinq ans au moment de l'enquête. Pour ce qui est du nombre d'employés, 72,0\% des entreprises ont moins de 50 employés et c'est le groupe des «moins de 10 employés» qui est le plus représenté, avec 32,0\% des répondants. Nous remarquons aussi que la catégorie qui regroupe les plus grandes entreprises (entre 100 et 200 employés) compte $16,0 \%$ des répondants.

En ce qui a trait aux secteurs d'activité, $68,0 \%$ des répondants sont actifs dans le grand secteur de la fabrication (SCIAN 31-33) et 32,0\% œuvrent dans le secteur des services scientifiques et techniques (SCIAN 541). Dans ce dernier groupe, nous retrouvons principalement des entreprises qui œuvrent dans l'industrie de la conception de systèmes informatiques et dans l'industrie de la recherche et développement en sciences physiques, de la vie et en génie. Cette dernière industrie regroupe essentiellement des entreprises du secteur des biotechnologies.

4. Au sujet de ce dernier point, précisons que l'information relative aux demandes de crédits d'impôts à la R-D est de nature confidentielle. Conséquemment, il est complexe d'identifier avec exactitude les entreprises qui ont demandé ces crédits d'impôts ou même de connaître leur nombre exact pendant la période 2002 à 2004. 
De plus, 60,0\% des entreprises avaient un chiffre d'affaires supérieur à un million de dollars en 2004 et 30,0\% un chiffre d'affaires inférieur à $500000 \$$. Le groupe des entreprises ayant un chiffre d'affaires inférieur à 250000 \$ est celui qui compte le plus d'entreprises (22,0\% des répondants), suivi de près par le groupe des entreprises ayant un chiffre d'affaires se situant entre 1 et 2,5 millions de dollars (20,0\%) qui occupe le second rang ex aquo avec le groupe des entreprises ayant un chiffre d'affaires supérieur à 10 millions de dollars. Par ailleurs, 84,0\% des répondants anticipent une croissance de leur chiffre d'affaires entre 2004 et 2007. En ce qui a trait aux exportations, $68,0 \%$ des entreprises vendent une partie de leur production hors des frontières canadiennes. Parmi les exportateurs, c'est le groupe qui comprend les entreprises qui vendent plus de 50,0\%, mais moins de 100,0\% de leur production à l'étranger qui est le plus important, avec $26,0 \%$ des répondants. Finalement, 8,0\% des entreprises réalisent l'ensemble de leurs ventes à l'étranger.

\section{TABLEAU 1}

\section{Caractéristiques des entreprises enquêtées}

\begin{tabular}{lcc}
\hline & \multicolumn{2}{c}{ Entreprises } \\
\hline & Fréquence & Pourcentage \\
\hline Période de création & 9 & \\
Avant 1980 & 10 & 18,0 \\
$1980-1990$ & 19 & 20,0 \\
$1990-2000$ & 12 & 38,0 \\
2000 et après & & 24,0 \\
\hline Personnel & 16 & \\
Moins de 10 employés & 14 & 32,0 \\
10 à 24 employés & 6 & 28,0 \\
25 à 49 employés & 6 & 12,0 \\
50 à 99 employés & 8 & 12,0 \\
100 à 200 employés & 35 & 16,0 \\
Croissance prévue du personnel d'ici 2007 & & 70,0 \\
\hline Secteurs d'activité & 34 & 68,0 \\
Secteur de la fabrication (SCIAN 31-33) & 16 & 32,0 \\
Services scientifiques et techniques (SCIAN 541) & & \\
\hline Chiffre d'affaires & 11 & 22,0 \\
Moins de 250 000 \$ & 9 & 18,0 \\
250 000 \$ à 1 M\$ & 15 & 30,0 \\
1 M \$ à 5 M \$ & 15 & 30,0 \\
5 M $\$$ et plus & 42 & 84,0 \\
Croissance prévue du chiffre d'affaires d'ici 2007 & & \\
\hline
\end{tabular}


TABLEAU 1

Caractéristiques des entreprises enquêtées (suite)

\begin{tabular}{lll}
\hline & \multicolumn{2}{c}{ Entreprises } \\
\hline Exportation & Fréquence & Pourcentage \\
Aucune & 21 & \\
Moins de $25 \%$ & 5 & 42,0 \\
$25 \%$ à $50 \%$ & 7 & 10,0 \\
Plus de $50 \%$, mais moins de $100 \%$ & 13 & 14,0 \\
$100 \%$ & 4 & 26,0 \\
\hline
\end{tabular}

Source: Enquête Estrie 2005.

\section{Profil de la région de l'Estrie}

L'Estrie est située au sud-est de la province de Québec et s'étend sur 10195 km². Bordée au sud par les États américains du Vermont, du New Hampshire et du Maine, la région a une population d'environ 300000 habitants, dont près de la moitié vivent dans la région de Sherbrooke, principale ville de la région et cinquième agglomération de la province de Québec. Les autres habitants vivent dans l'une ou l'autre des six municipalités régionales de comté à caractère rural que compte l'Estrie.

Lorsque vient le temps de faire le portrait du système régional d'innovation estrien, une dualité fondamentale est observée. D'un côté, le secteur manufacturier occupe une place de premier plan:24,1\% $\%$ des emplois y étaient associés en 2001 et la majorité des 1141 entreprises manufacturières de la région sont des PME actives dans des secteurs de faible ou moyenne-faible intensité technologique. Du côté de l'innovation, nous constatons que les entreprises en Estrie affichent des performances inférieures à la moyenne québécoise : la DIRDE ${ }^{6}$ par habitant est 7,5 fois moins importante que la moyenne provinciale, l'entreprise estrienne active en R-D dépense en moyenne 2,7 fois moins que la moyenne provinciale. Notons aussi que les exportations jouent un rôle prépondérant dans l'économie régionale: en 2003,43 \% des entreprises manufacturières vendaient une partie de leur production hors des frontières canadiennes (Institut de la statistique du Québec, 2004).

De l'autre côté, il y a un pôle universitaire excessivement dynamique doté de centres de recherche d'envergure internationale fréquentés par plus de 20000 étudiants. Ce pôle universitaire regroupe neuf institutions d'enseignement supérieur et de recherche de la région, dont l'Université

5. La moyenne au Québec est de 16,6\%.

6. Dépenses de R-D intra-muros des entreprises.

Revue internationale P.M.E., vol. 20, nº 1, 2007 
de Sherbrooke, l'Université Bishop et le Centre hospitalier universitaire de Sherbrooke. D'après des statistiques de l'Institut de la statistique du Québec (2004), la région de l'Estrie affiche la plus forte concentration de professeurschercheurs universitaires par 10000 habitants au Québec. De même, en 2001, parmi les 4500 diplômés, près de 800 détenaient une maîtrise ou un doctorat. En ce qui a trait à la recherche qui est réalisée en Estrie, l'examen des publications en sciences naturelles et génie révèle que la région se classe au troisième rang dans la province du Québec, derrière Montréal et Québec.

\section{Résultats empiriques}

\subsection{Activités d'innovation}

Cette section relève les pratiques innovantes des entreprises qui ont participé à l'enquête. Le tableau 2 présente un portrait descriptif de différents déterminants de l'innovation, dont les stratégies d'innovation, les dépenses de R-D, le type de personnel impliqué en R-D, les principales sources d'information et les types d'innovation réalisés dans l'entreprise.

\section{TABLEAU 2}

\section{Activités d'innovation dans les entreprises enquêtées}

\begin{tabular}{|c|c|c|}
\hline \multirow[b]{2}{*}{ Indicateurs } & \multicolumn{2}{|c|}{ Entreprises } \\
\hline & Fréquence & Pourcentage \\
\hline $\begin{array}{l}\text { Stratégies d'innovation jugées importantes pour le } \\
\text { succès des entreprises }\end{array}$ & 45 & 90,0 \\
\hline R-D réalisée dans l'entreprise & 16 & 32,0 \\
\hline R-D réalisée hors de l'entreprise & 30 & 60,0 \\
\hline Acquisition de technologies et d'équipements & 18 & 36,0 \\
\hline Acquisition de logiciels spécialisés & 12 & 24,0 \\
\hline Achat de licences/droits d'exploitation & 32 & 64,0 \\
\hline Formation du personnel affecté à l'innovation & 27 & 54,0 \\
\hline Embauche de personnel affecté à l'innovation & 12 & 24,0 \\
\hline Ingénierie inverse & 22 & 44,0 \\
\hline $\begin{array}{l}\text { Implantation de nouveaux modes d'organisation } \\
\text { de la production }\end{array}$ & & \\
\hline Dépenses de R-D par rapport au chiffre d'affaires & & \\
\hline Moins de $0,5 \%$ & 8 & 16,0 \\
\hline $0,5 \%$ à $1,5 \%$ & 6 & 12,0 \\
\hline $1,5 \%$ à $5 \%$ & 10 & 20,0 \\
\hline $5 \%$ à $10 \%$ & 4 & 8,0 \\
\hline $10 \%$ et plus & 19 & 38,0 \\
\hline
\end{tabular}


TABLEAU 2

Activités d'innovation dans les entreprises enquêtées (suite)

\begin{tabular}{lcc}
\hline & \multicolumn{2}{c}{ Entreprises } \\
\hline Indicateurs & Fréquence & Pourcentage \\
\hline Type de personnel impliqué en R-D & 35 & 70,0 \\
Technicien & 28 & 56,0 \\
Ingénieur & 17 & 34,0 \\
Scientifique & 11 & 22,0 \\
Marketing/vente & 6 & 12,0 \\
Autre & & \\
Principales sources d'information & 41 & 82,0 \\
Clients & 29 & 58,0 \\
Fournisseurs & 18 & 36,0 \\
Concurrents & 19 & 38,0 \\
Universités & 9 & 18,0 \\
Centres de recherche publics & 9 & 18,0 \\
Centres de transfert technologique & 24 & 48,0 \\
Foires, expositions et congrès & & \\
Innovation de produit/service & 40 & 80,0 \\
Au moins un nouveau produit/service & 14 & 28,0 \\
Première mondiale & 9 & 18,0 \\
Première canadienne & 21 & 42,0 \\
Première québécoise & & \\
\hline Innovation de procédé & 27 & 54,0 \\
Au moins un nouveau procédé & 16 & 16,0 \\
Première mondiale & 6 & 6,0 \\
Première canadienne & 2 & 2,0 \\
Première québécoise & &
\end{tabular}

Source: Enquête Estrie 2005.

Dans le cas des stratégies d'innovation, nous avons demandé aux entreprises d'indiquer, parmi une liste de stratégies, celles ayant de l'importance pour la réalisation de leurs activités d'innovation. Les résultats du tableau montrent que la R-D réalisée dans l'entreprise est la stratégie la plus souvent citée (90\%), suivie de la formation du personnel affecté à l'innovation (64 \%) puis de l'acquisition de technologies et d'équipement, activité jugée importante par $60 \%$ des répondants. Parmi les stratégies jugées comme étant peu importantes se trouvent l'ingénierie inverse $(24,0 \%)$, l'achat de licences et de droits d'exploitation (24,0\%), la R-D réalisée hors de l'établissement $(32,0 \%)$, de même que l'acquisition de logiciels spécialisés (36,0\%).

L'enquête a cherché à obtenir des informations sur la part du chiffre d'affaires des entreprises qui était consacrée à la R-D. Les résultats du 
tableau 3 révèlent que 38,0 \% des entreprises ont consacré plus de 10,0 \% de leur chiffre d'affaires à la R-D; c'est au sein de ce groupe que se trouve le plus grand nombre de répondants. Les 20,0\% d'entreprises ayant consacré entre $1,5 \%$ et 5,0 \% de leur chiffre d'affaires à la R-D occupent le deuxième rang, devant les entreprises ayant consacré moins de $0,5 \%$ de leur chiffre d'affaires à la R-D. L'examen du type de personnel engagé dans les activités de R-D permet de constater que ce sont principalement les techniciens et les ingénieurs qui contribuent aux activités de R-D, comparativement au personnel scientifique, de marketing/vente et aux autres employés qualifiés ${ }^{7}$.

L'enquête s'est également intéressée aux sources d'information utilisées par les entreprises lors de leurs activités d'innovation. Les résultats présentés au tableau 2 illustrent que les sources technologiques sont moins fréquemment utilisées que les sources de marché. Ainsi, les clients et les fournisseurs, mentionnés respectivement par 82,0\% et 58,0\% des entreprises, occupent les deux premières positions, alors que l'université $(38,0 \%)$, les centres de recherche publics et les centres de transfert technologique (18,0\% chacun) sont des sources d'informations moins utilisées par les entreprises de l'enquête. Ces données corroborent le fait que, parmi les sources externes utilisées par les entreprises, les clients et les fournisseurs sont celles les plus fréquemment utilisés par les entreprises. Ce sont d'ailleurs ces sources qui exercent l'impact marginal le plus élevé sur la probabilité des entreprises à innover (Amara et Landry, 2005; Cooke, Boekholt et Tödtling, 2000;Tödtling et Kaufmann, 1999).

En ce qui concerne les innovations réalisées, nous avons demandé aux entreprises d'indiquer si elles ont introduit sur le marché des produits et services et des procédés nouveaux ou significativement améliorés au cours entre 2002 et $2004^{8}$. À ce sujet, les résultats du tableau 2 démontrent que l'innovation de produit et service est plus fréquente que l'innovation

7. Au sein du groupe «autre employé», nous retrouvons tant du personnel associé à la production (outilleurs, opérateurs, machinistes, soudeurs, etc.) que du personnel administratif (contrôleur, adjointe administrative, etc.) que des professionnels (programmeurs, médecins, infirmières, etc.).

8. Dans le cadre de l'enquête, une innovation est «soit un produit (bien ou service) nouveau ou modifié significativement introduit sur le marché, soit l'introduction dans l'entreprise d'un procédé nouveau ou modifié significativement». Les innovateurs de produits sont donc des entreprises ayant déclaré avoir introduit, au cours des dernières années précédant l'enquête « un bien ou un service qui est nouveau ou modifié significativement au regard de ses caractéristiques fondamentales, de ses spécifications techniques, des logiciels incorporés ou de tout autre composant immatériel, de l'utilisation prévue ou de la facilité d'usage ». Les innovateurs de procédés sont des entreprises ayant déclaré avoir adopté (développé en interne ou non), au cours des trois dernières années précédant l'enquête, «soit une technologie de production nouvelle ou modifiée significativement, soit une méthode nouvelle ou modifiée significativement de fourniture de services ou de livraison de produits », et ce, avec un résultat significatif sur le niveau, la qualité ou les coûts de production. Une entreprise innovante a introduit soit une innovation de produit, soit une innovation de procédé, soit les deux, au cours des trois années précédant l'enquête. 
de procédé. En effet, alors que 80,0\% des répondants ont indiqué avoir introduit, pendant la période 2002 à 2004, au moins un nouveau produit ou service, seulement $54,0 \%$ des répondants ont déclaré avoir introduit au moins un nouveau procédé. Notons aussi que, parmi les répondants, 28,0\% ont précisé que leur nouveau produit (ou service) le plus innovateur constituait une première mondiale, $18,0 \%$, une première canadienne et $42,0 \%$, une première québécoise. De plus, $16,0 \%$ des répondants ont indiqué considérer le procédé le plus innovateur introduit par leur entreprise entre 2002 et 2004 comme une première mondiale, $6,0 \%$, comme une première canadienne et 2,0\%, comme une première québécoise.

\subsection{Réseaux d'innovation, proximité et le rôle de la région}

Cette section présente les résultats sur les réseaux d'innovation mis en œuvre par les entreprises. Au cours de l'enquête, nous avons demandé aux entreprises d'indiquer si elles ont réalisé des collaborations dans le cadre de leurs activités d'innovation et, le cas échéant, les types de partenaires avec lesquels elles avaient collaboré. Le tableau 3 résume les résultats.

TABLEAU 3

\section{Entreprises ayant des collaborations et types de collaborateurs}

\begin{tabular}{lcc}
\hline & \multicolumn{2}{c}{ Entreprises } \\
\hline & Nombre & $\%$ \\
\hline Entreprises ayant au moins une collaboration & 27 & 54,0 \\
Types de collaborateurs & 18 & 36,0 \\
Clients & 12 & 24,0 \\
Fournisseurs & 1 & 2,0 \\
Concurrents & 12 & 24,0 \\
Universités & 2 & 4,0 \\
Cégeps et collèges & 7 & 14,0 \\
Centres de recherche & 12 & 24,0 \\
Firmes d'experts-conseils & 2 & 4,0 \\
Centres de recherche privés & 4 & 8,0 \\
Centres de transfert technologiques &
\end{tabular}

Le tableau 3 permet de constater que plus de la moitié des entreprises $(54,0 \%)$ ont collaboré dans le cadre d'activités innovantes. Les collaborateurs de marché figurent parmi les types de collaborations les plus fréquemment développées par les entreprises de l'enquête. Ainsi, les collaborations avec les clients, qui sont menées par 36,0\% des entreprises, sont les plus 
fréquentes alors que les collaborations avec les fournisseurs (24,0\% des entreprises) arrivent au second rang, ex жеquo avec les firmes d'experts-conseils et l'université, des partenaires technologiques. Les entreprises de l'Estrie font moins fréquemment appel aux autres types de collaborateurs pour mener à bien leurs activités d'innovation.

L'analyse des réseaux d'innovation doit aussi tenir compte de l'origine géographique des collaborateurs. En effet, les études empiriques récentes ont démontré que l'innovation est un processus fondé sur des relations de proximité et qu'elle est intimement liée et stimulée par la proximité géographique qui permet d'accroître la capacité interactive des formes d'apprentissage entre l'entreprise innovante et les apports externes qui lui sont nécessaires lors du processus d'innovation.

Afin d'apprécier le rôle de la région et de la proximité, dans l'enquête, nous avons demandé aux entreprises ayant déclaré des collaborations à l'innovation, d'établir la provenance de chacun des types de partenaires avec lesquels elles avaient collaboré. Le tableau 4, en plus de présenter le nombre total de collaborations, détaille la provenance géographique des types de collaborateurs à l'innovation (régionale, provinciale, nationale et internationale).

TABLEAU 4

\section{Collaborations selon le type de collaborateurs et la provenance géographique $(n=27)$}

\begin{tabular}{|c|c|c|c|c|c|c|}
\hline \multirow{3}{*}{$\begin{array}{l}\text { Type de } \\
\text { collaborateur }\end{array}$} & \multicolumn{4}{|c|}{ Provenance géographique des collaborateurs } & \multirow{2}{*}{\multicolumn{2}{|c|}{$\begin{array}{l}\text { Ensemble des } \\
\text { collaborations }\end{array}$}} \\
\hline & \multirow{2}{*}{ Estrie } & \multirow{2}{*}{$\begin{array}{l}\text { Ailleurs au } \\
\text { Québec }\end{array}$} & \multirow{2}{*}{$\begin{array}{l}\text { Ailleurs au } \\
\text { Canada }\end{array}$} & \multirow{2}{*}{$\begin{array}{c}\text { Autres } \\
\text { pays }\end{array}$} & & \\
\hline & & & & & $n$ & $\%$ \\
\hline Clients & 9 & 10 & 6 & 10 & 35 & 33,3 \\
\hline Fournisseurs & 6 & 4 & 1 & 5 & 16 & 15,2 \\
\hline Concurrents & 0 & 0 & 0 & 1 & 1 & 1,0 \\
\hline Universités & 10 & 5 & 2 & 3 & 20 & 19,0 \\
\hline Cégeps et collèges & 0 & 2 & 0 & 0 & 2 & 1,9 \\
\hline Centres de recherche & 3 & 4 & 3 & 0 & 10 & 9,5 \\
\hline $\begin{array}{l}\text { Firmes } \\
\text { d'experts-conseils }\end{array}$ & 7 & 6 & 0 & 1 & 14 & 13,3 \\
\hline $\begin{array}{l}\text { Centres de } \\
\text { recherche privés }\end{array}$ & 2 & 0 & 1 & 0 & 3 & 2,9 \\
\hline $\begin{array}{l}\text { Centres de transfert } \\
\text { technologique }\end{array}$ & 0 & 4 & 0 & 0 & 4 & 3,8 \\
\hline Total & 37 & 35 & 13 & 20 & 105 & 100,0 \\
\hline
\end{tabular}

Source: Enquête Estrie 2005. 
Le tableau 4 présente la provenance géographique des collaborations à l'innovation et le nombre total de collaborations réalisées. Parmi les 50 entreprises de notre échantillon, 27 entreprises ont collaboré dans le cadre de leurs activités d'innovation. Elles ont initié 105 collaborations distinctes, soit une moyenne de 3,9 collaborations par entreprise. Ces collaborations se sont déroulées avec des partenaires localisés à différents niveaux géographiques. Pour innover, les entreprises estriennes combinent donc les ressources et expertises disponibles au sein du système régional d'innovation et à l'extérieur de celui-ci. En effet, bien que 35,2\% des collaborations soient réalisées à l'échelle régionale, la région n'est pas le seul lieu où les collaborations s'observent. Ainsi, 64,8\% des collaborations sont établies avec des partenaires situés hors de la région, dont $20 \%$ avec des partenaires internationaux.

La plupart des entreprises maintiennent des collaborations à différents niveaux géographiques et collaborent, en moyenne, avec des partenaires localisés dans 2,2 niveaux géographiques différents. Ce résultat illustre bien que l'Estrie est une région au carrefour de plusieurs réseaux, ce qui s'explique notamment par sa proximité des grands marchés québécois et du nord-est américain. De plus, ces résultats corroborent les travaux récents sur les réseaux d'innovation qui démontrent l'importance pour les entreprises innovantes d'avoir, non seulement accès, mais également la possibilité de transférer et partager des connaissances du plan régional jusqu'au plan international (Doloreux, 2004a; Simmie, 2003; Britton, 2004).

En analysant les différents types de partenaires en fonction de l'échelle géographique, les données présentées au tableau 4 permettent de constater les faits saillants suivants:

- À l'échelle régionale et provinciale, deux types de collaboration sont particulièrement importants, soit les collaborations de marchés avec les clients et fournisseurs et les collaborations avec certaines sources axées sur le développement technologique (universités et firmes d'experts-conseils). Ces résultats reflètent la dualité qui caractérise l'économie de l'Estrie. En effet, d'un côté, il y a des entreprises qui évoluent dans des secteurs à faible intensité technologique, ce qui laisse entrevoir que les collaborations de ces entreprises seront réalisées avec les clients et fournisseurs avec comme objectif principal d'améliorer le développement de produits et de développer de nouvelles méthodes de marketing et de distribution. De l'autre côté, il y a des PME génératrices de technologies qui ont des réseaux axés sur le développement technologique et sont très fortement liées aux 
établissements du pôle technologique estrien. Nous devons signaler qu'une partie de ces entreprises sont essaimées de l'Université de Sherbrooke.

- À l'échelle nationale, les entreprises font peu appel aux collaborateurs provenant du système national d'innovation. Pour un segment d'entreprises, des collaborations à l'échelle nationale sont observées, notamment avec les clients et les centres de recherche. Ces résultats peuvent s'expliquer par la proximité des deux principales régions métropolitaines au Québec, Montréal et la ville de Québec.

- À l'échelle internationale, les entreprises coopèrent plus intensivement avec les clients et les fournisseurs; il est rare que les entreprises collaborent à l'échelle internationale avec les concurrents, les institutions de R-D et les centres de recherche. Les coopérations marchandes avec les clients et fournisseurs à l'extérieur du Canada permettent à l'entreprise l'accélération des processus d'internationalisation à l'échelle globale, plus particulièrement en ce qui a trait aux marchés. En effet, les entreprises enquêtées sont reliées à l'ensemble de la collectivité économique internationale et beaucoup d'entre elles exportent à l'extérieur du Canada.

\section{Conclusion}

L'article cherchait à établir les pratiques innovantes des PME en Estrie et à analyser les formes de collaboration de provenance régionale, nationale et internationale. Au cœur de cet article se situe la question du rôle de la proximité et des régions dans les réseaux d'innovation des PME.

Pour répondre à ces questions, nous avons insisté en premier lieu sur la dimension régionale de l'innovation et défini l'innovation comme étant le résultat d'un processus systémique tributaire de l'apprentissage interactif des externalités régionales. Cette définition souligne l'importance de deux facteurs importants: l'influence de l'environnement culturel, économique et institutionnel sur les activités innovantes et l'effet de la proximité géographique des acteurs sur la transmission de l'information stratégique et du savoir.

L'étude des pratiques innovantes et des réseaux d'innovation utilisés par des entreprises de l'Estrie a permis d'appuyer les études de Landry (1998) qui ont démontré que les entreprises dans cette région sont innovantes et bénéficient de leurs liens avec d'autres entreprises. Les collaborations élaborées avec les sources externes de marché (clients et fournisseurs) sont les plus fréquentes et sont celles qui exercent l'impact le plus élevé sur la probabilité 
des entreprises à innover, supportant les observations de Von Hippel (1988) et Lundvall (1992). De plus, les résultats concernant la provenance géographique des collaborations ont permis de mettre en valeur à la fois l'importance des ressources régionales et des réseaux plus larges. Par ailleurs, les entreprises enquêtées coopèrent plus intensivement avec les institutions de R-D et les firmes de services spécialisés du système régional d'innovation. Aussi, les entreprises n'ont pas ou peu établi de liens de coopération avec certains acteurs régionaux, notamment les concurrents, les cégeps et collèges, les laboratoires publics de R-D et les centres de transfert technologique.

Les résultats montrent aussi l'importance relative des réseaux plus larges. Ces réseaux restent marchands, en général, mais des liens avec d'autres entreprises et institutions ne sont pas exclus. Les entreprises bénéficient de leurs liens avec les clients et fournisseurs internationaux pour innover. En effet, les réseaux plus larges confèrent des avantages dans la création d'innovation et de connaissances en intégrant l'entreprise dans des environnements multiples. Cela lui permet ainsi d'être exposée et de bénéficier d'un éventail de compétences plus large provenant à la fois du plan local et du plan global, le tout dans le but d'obtenir et de maintenir un avantage concurrentiel.

Ceci dit, le rôle du milieu est certes important dans les processus d'innovation des entreprises, mais la réalité que recouvre cette importance demeure questionnable, du moins, selon les résultats de l'enquête auprès des PME de l'Estrie. Les résultats démontrent l'importance non seulement des collaborations et processus d'apprentissage localisés mais aussi de ceux qui sont plus diffus spatialement. Ainsi, les réseaux d'innovation des entreprises, même s'ils sont en partie liés à la région dans laquelle elles se localisent, le sont tout autant avec le monde extérieur. Dans ce cas, il est impératif pour mieux comprendre la nature et l'étendue des réseaux d'innovation des PME d'étudier les «espaces» de relations des entreprises, et ce, en considérant que l'innovation évolue dans un système global composé de différents soussystèmes dans lesquels évolue l'entreprise, par exemple, le système régional d'innovation et le système national d'innovation. Interroger davantage les entreprises sur l'étendue de leurs liens avec d'autres sources externes permettrait une meilleure compréhension de ce phénomène et constituerait un apport important pour des voies futures de recherche sur l'innovation, les entreprises et le rôle joué par la proximité et la région. 


\section{Bibliographie}

Amable, B., R. Barré et R. Boyer Amable (1997), Les systèmes d'innovation à l'ère de la globalisation, Paris, Économica.

AmARA, N. et R. LANDRY (2005), «Sources of information as determinants of novelty of innovation in manufacturing firms : evidence from the 1999 statistics Canada innovation survey», Technovation, vol. 25, p. 245-259.

Asheim, B.T. et M.S. Gertler (2005), « Regional innovation systems and the geographical foundations of innovation», dans J. Fagerberg, D. Mowery et R. Nelson (dir.), Oxford Handbook of Innovation, Londres, Oxford University Press, p. 291-317.

Bitard, P. et D. Doloreux (2006), «Configurations régionales des dynamiques d'innovation en Suède: une analyse à partir de l'enquête communautaire de l'innovation III», dans J.L. Klein et C. Tardif (dir.), Entre réseaux et systèmes: les nouveaux espaces régionaux, Rimouski, Éditions du CRDT, p. 129-155.

BRITTON, J.N.H. (2003), «Network structure of an industrial cluster: electronics in Toronto», Environment and Planning A, vol. 35, p. 983-1006.

BRitTon, J.N.H. (2004), «High technology localization and extra-regional networks », Entrepreneurship and Regional Development, vol. 16, p. 369-390.

Bunnell, T.G. et N.M. Coe (2001), «Spaces and scales of innovation», Progress in Human Geography, vol. 25, p. 569-589.

Camagni, R., D. Maillat et A. Matteaccioli (2004), Ressources naturelles et culturelles, milieux et développement local, Neuchâtel, Éditions EDES.

Cooke, P., P. Boekholt et F. Tödtling (2000), The Governance of Innovation in Europe: Regional Perspectives on Global Competitiveness, Londres, Pinter.

DeBresson, C. et F. Amesse (1991), «Networks of innovators : a review and introduction to the issue», Research Policy, vol. 20, p. 363-379.

DiEZ, J.R. (2000), «Innovative networks in manufacturing: some empirical evidence from the metropolitan area of Barcelona», Technovation, vol. 20, p.139-150.

Doloreux, D. (2003), «Regional innovation systems in the periphery: the case of the Beauce in Québec (Canada)», International Journal of Innovation Management, vol. 7, p. 67-94.

Doloreux, D. (2004a), «Regional innovation systems in Canada: a comparative study», Regional Studies, vol. 38, p. 479-492.

Doloreux, D. (2004b), «Regional networks of small and medium sized enterprises: evidence from the metropolitan area of Ottawa in Canada», European Planning Studies, vol. 12, p. 173-189.

Edquist, C. (1997), Systems of Innovation: Technologies, Institutions and Organizations, Londres, Pinter.

Edquist, C., M.-L. EriKsson et H. SJÖREN (2000), «Collaboration in product innovation in the East-Gothia regional innovation system », Enterprise and Innovation Management Studies, vol. 1, p. 37-56.

Revue internationale P.M.E., vol. 20, nº 1, 2007 
Edquist, C., M.-L. Eriksson et H. SJöREN (2002), « Characteristics of collaborations in product innovation in the regional innovation system of East-Gothia», European Planning Studies, vol. 10, p. 563-581.

Fischer, M. (2001), «Innovation, knowledge creation and systems of innovation», The Annals of Regional Science, vol. 35, p. 199-216.

FreEL, M.S. (2002), «On regional systems of innovation: illustrations from the West Midlands», Environment and Planning C-Government and Policy, vol. 20, p. 633-654.

Freel, M.S. (2003), «Sectoral patterns of small firm innovation, networking and proximity», Research Policy, vol. 32, p. 751-770.

Grabher, G. (1993), The Embedded Firm: On the Socio-Economics of Industrial Networks, Londres, Routledge.

Gregerson, B. et B. Johnsson (1997), «Learning economies, innovation systems and European integration», Regional Studies, vol. 4, p. 479-490.

HenRy, N. et S. Pinch (2000), «Specialising knowledge: placing the knowledge community of Motor Sport Valley», Geoforum, vol. 31, p. 191-208.

Howells, J. (1999), «Regional innovation systems? », dans D. Archibugi, J. Howells et J. Michie (dir.), Innovation Policy in a Global Economy, Cambridge, Cambridge University Press, p. 67-93.

Institut DE LA STATISTIQUe DU QuéBeC (2004), Tableau de bord des systèmes régionaux d'innovation du Québec, Québec, Gouvernement du Québec.

Koschatzky, K., M. Kulicke et A. Zenker (2001), Innovation Networks: Concept and Challenges in the European Perspective, Heidelberg, Physica-Verlag.

LANDRY, R. (1998), L'innovation de produits et de procédés de fabrication dans les entreprises manufacturières de l'Estrie, Sherbrooke, Groupe d'action pour l'avancement technologique de l'Estrie.

LARsson, S. et A. MALMBERG (1999), «Innovations, competitiveness and local embeddedness: a study of machinery producers in Sweden », Geografiska Annaler, vol. 81, p. 1-18.

Lorenzen, M. (1998), Specialisation and Localised Learning, Copenhage, Copenhagen Business School.

Lundvald, B.-A. (1992), National Systems of Innovation: Towards a Theory of Innovation and Interactive Learning, Londres, Pinter.

Lyons, D. (2000), «Embeddedness, milieu, and innovation among high-tech firms », Environment and Planning A, vol. 32, $\mathrm{n}^{\circ}$ 5, p. 891-908.

MacKinnon, D., K. Chapman et A. Cumbers (2004), «Networking, trust and embeddedness amongst SMEs in the Aberdeen oil complex», Entrepreneurship and Regional Development, vol.16, p. 87-106.

MacKinnon, D., A. Cumbers et K. Chapman (2002), «Learning, innovation and regional development: a critical appraisal», Progress in Human Geography, vol. 26, p. 293-311.

Revue internationale P.M.E., vol. 20, nº 1, 2007 
Maillat, D. et L. KéBIR (2001), «Conditions-cadres et compétitivité des régions : une relecture», Revue canadienne des sciences régionales, vol. 24, p. 41-56.

Malmberg, A. et P. Maskell (1997), «Towards an explanation of regional specialization and industry agglomeration», European Planning Studies, vol. 5, n ${ }^{\circ} 1$, p. 25-41.

MASKell, P. (2001), «Towards a knowledge-based theory of the geographical cluster», Industrial and Corporate Change, vol. 10, p. 921-943.

Maskell, P. et A. Malmberg (1999), «Localised learning and industrial competitiveness », Cambridge Journal of Economics, vol. 23, p. 167-186.

Morgan, K. (1997), «The learning region: institutions, innovation and regional renewal », Regional Studies, vol. 31, p. 491-503.

ORganisation de CoOpÉRATION ET DE DÉVELOPPEMENT ÉCONOMIQUes - OCDE (1997), Oslo Manual, Paris.

Porter, M.E. (1998), «Clusters and the new economics in competition», Harvard Business Review, vol. 76, p. 77-90.

Rogers, M. (2004), «Networks, firm size and innovation », Small Business Economics, vol. 22, p. 141-153.

Romijn, H. et M. Albaladejo (2002), «Determinants of innovation capability in small electronics and software firms in southeast England», Research Policy, vol. 31, p. 1053-1067.

Simmie, J. (2001), Innovative Cities, Londres, Spon.

Simmie, J. (2003), «Innovation and urban regions as national and international nodes for the transfer and sharing of knowledge», Regional Studies, vol. 37, p. 607-620.

STERnBerG, R. (2000), «Innovation networks and regional development - evidence from the European regional innovation survey (ERIS): theoretical concepts, methodological approach, empirical basis and introduction to the theme issue», European Planning Studies, vol. 8, p. 389-407.

Storper, M. (1997), The Regional World, New York, The Guillford Press.

SuArlez-Villa, L. et W. Walrod (1997), «Operational strategy, R\&D and the intrametropolitan clustering in a polycentric structure», Urban Studies, vol. 34, p. 1343-1380.

TiJssen, R.J.W. (1998), «Quantitative assessment of large heterogeneous R\&D networks : the case of process engineering in the Netherlands », Research Policy, vol. 26, p. 791-809.

TöDtLing, F. et A. Kaufmann (1999), «Innovation systems in regions of Europe: a comparative perspective», European Planning Studies, vol. 7, p. 699-717.

TöDtLing, F. et A. Kaufmann (2001), «The role of the region for innovation activities of SMEs », European Urban and Regional Studies, vol. 8, p. 203-215.

Von Hippel, E. (1988), The Sources of Innovation, Cambridge, MIT Press.

Revue internationale P.M.E., vol. 20, nº 1, 2007 